\title{
Two-Dimensional Dipon Forward Modeling of High-Power Induced Polarization Mid-Stair Geological Exploration
}

\author{
Wang Liang*, Zhang Shaodong, Lv Zhibin, Zhu Jiejun \\ Qinghai Qaidam Comprehensive Geological and Mineral Exploration Institute, Qinghai, China \\ *corresponding athor
}

Keywords: Induced polarization(ip) effect, Two-dimensional magnetotelluric, Cole-cole model, Data space

\begin{abstract}
Among the geophysical exploration methods, conventional magnetotelluric methods have extensive and in-depth applications in many fields such as deep crustal structure and petroleum exploration. In this paper, by using the Cole-Cole model to introduce the complex resistivity into the Maxwell equation, the feasibility of extracting the four parameters of the ColeCole model from the observation data is explored, and finally the programming realizes the magnetotelluric two-dimensional tilter positive performance research. The staggered grid finite difference method is used for forward modeling, the conductivity in Maxwell equation is replaced with complex conductivity through Cole-Cole model, and Maxwell equation is discretized based on staggered sampling grid division and combined with boundary conditions to obtain the electric field value satisfied Linear equations. This paper analyzes the influence of the parameters of the ColeCole model on the polarizer through the forward simulation of two polarizers in a uniform halfspace. The forward modeling results of the model show that the existence of the IP effect will increase the apparent resistivity value, and different IP parameters have different effects on the apparent resistivity. Among them, the polarizability has the greatest influence on the apparent resistivity; the greater the polarizability is , the greater the apparent resistivity.
\end{abstract}

\section{Introduction}

Geophysics is a discipline based on physical knowledge for detecting underground space and surrounding space[1]. Electromagnetic exploration is one of the important subdisciplines. The basis of the physical properties used is the dielectric properties, electrical conductivity differences and magnetization properties of rock minerals[2]. We can use geophysical related instruments to detect the distribution characteristics of the fields corresponding to these differences in underground physical properties, and further use forward and inverse algorithms to infer the underground spatial distribution. MT is a frequency domain electromagnetic method, which contains rich frequency information from low to high, corresponding to the corresponding skin depth, that is to say, it can detect underground information at various depths to meet the requirements of depth sounding. The data receiving method is to collect mutually perpendicular electromagnetic field components on the ground. The main physical basis is the difference in electrical conductivity between rocks and minerals. With continuous development, the application fields are becoming more and more extensive.

Generally speaking, MT only reflects the conductivity information of rock and ore without considering the IP anomaly. The existence of IP anomalies in actual media is inevitable under certain circumstances, so the study of the MT three-dimensional algorithm that considers the IP effect can also consider the IP characteristics and conductive properties of rock and ore, and conduct research in a unified way. In this way, more physical parameters can be obtained by inversion, and it is closer to the real underground media, which further expands the scope of application and exploration effects[3].

In general, the introduction of the induced polarization effect in the magnetotelluric method can realize that only the forward and inverse theoretical method is updated. On the basis of not needing 
to increase the amount of field data collection, it is possible to obtain richer and more suitable electrical sexual parameters[4]. The electrical conductivity of the underground medium, the induced polarization effect, the uniformity of the conductive particles in the rock and ore, and the connectivity can all be evaluated by these parameters. At the same time, the role of IP information in the fields of hydrogeological engineering and oil and gas exploration is also very huge. If IP information can be extracted from MT, its application range can be greatly increased. Magnetotelluric sounding is a frequency-domain electromagnetic method that uses natural electromagnetic fields to study deep geological structures. This method does not require artificial field sources to be established[5]. It has the characteristics of portable equipment and convenient work. It is widely used to study the crust and upper earth. Slow geological structure, oil and gas or geothermal exploration, etc. At present, scholars at home and abroad have done a lot of work on magnetotelluric forward modeling. In the early 1940s, Cole KS and Cole RH proposed a formula to describe the induced polarization effect. In the 1970s, it was proposed to combine the finite element method with the magnetotelluric numerical simulation, and made a comprehensive description of how to use the finite element to simulate the magnetotelluric field[5].

Cui, X[6] et al. used the results of field experiments to preliminarily confirmed the feasibility of passive sources used for excitation polarization under certain conditions; Li, Y [7] et al. used natural field sources for excitation under certain conditions. The feasibility of the chemical method is verified, and the electromagnetic effect and the IP effect are considered together. The experimental results show that at a certain frequency, the apparent resistivity with the IP effect is higher than the apparent resistance without the IP effect. However, we believes that the existence of the induced polarization effect will reduce the apparent resistivity. The author uses the finite element method to forward simulations of different types of polarizers in non-polarized uniform half-spaces to analyze the influence of different polarizabilities on the polarizers, and obtain the increase in apparent resistivity in conclusion.

\section{Forward Theory}

In the theoretical study of the natural field source induced polarization method, there are two common ideas: one is to separate the induced polarization effect from the induction effect by calculating the ratio of the video scatter rate or the potential difference; the other is to use the ColeCole model to describe the resulting response law. This article adopts the second idea.

\subsection{The Equation Satisfied When the Magnetotelluric Field Considers the Induced Polarization Effect}

The wave equation of electromagnetic wave describes the change law of electric or magnetic field with time and space. The wave equation of harmonic field is also called Helmholtz equation, which is often used to describe the electric field and magnetic field in magnetotelluric[8]. The finite element method is used to solve the unknown electric and magnetic fields in the equation, so as to calculate the apparent resistivity and phase under the model to achieve the purpose of forward modeling of magnetotelluric under the two-dimensional geoelectric condition.

Assuming that the geoelectric model is two-dimensional, the $\mathrm{X}$-axis and $\mathrm{Y}$-axis are taken as the survey line direction, the Z-axis is vertically downward, and the survey line direction is perpendicular to the strike. At the same time, the negative time harmonic factor $\varepsilon$ is taken, and the magnetic permeability in the earth is equal to vacuum permeability, the Helmholtz equation under the two-dimensional magnetotelluric response can be derived from the Maxwell equations[9]:

$$
\begin{aligned}
& \frac{d}{d y}\left(\frac{d \vec{\Sigma}}{d y} \frac{1}{j \vartheta \mu}\right)+\frac{d}{d z}\left(\frac{d \vec{\Sigma}}{d z} \frac{1}{j \vartheta \mu}\right)+(\eta-j \vartheta \mu) \vec{\Sigma}=0 \\
& \frac{d}{d y}\left(\frac{d \vec{\Gamma}}{d y} \frac{1}{\eta-j \vartheta \mu}\right)+\frac{d}{d z}\left(\frac{d \vec{\Gamma}}{d z} \frac{1}{\eta-j \vartheta \mu}\right)+j \vartheta \mu \vec{\Gamma}=0
\end{aligned}
$$


Where: $\vec{\Gamma}$ and $\vec{\Sigma}$ are the total electric field and the total magnetic field respectively; $\eta$ is the conductivity of the medium; $\vartheta$ is the angular frequency; $\mu$ is the magnetic permeability. In the Cartesian coordinate system:

$$
\Theta=\frac{d}{d y} \vec{\Sigma}_{x}+\frac{d}{d z} \vec{\Sigma}_{z}
$$

In order to overcome the contradiction between precision and workload in the selection of grid size and element size, others adopted a non-uniform cross-symmetrical grid. The central part of the grid has small elements and dense nodes, and the edge elements are large and sparse. The cells are gradually enlarged from the center to the edge to ensure that the grid has sufficient size, and the complex parts of the geoelectrical section are located in the dense center of the grid to meet the conditions of uniform electrical properties in the cell and linear changes of the electromagnetic field potential function[10]. When solving the finite element method corresponding to the Helmholtz equation, the solution of the continuous field function is converted into the solution of the discrete function value at the node by using the cross-symmetric grid. Calculate the resistivity and phase of the visual application at each node by formulas (4), (5):

$$
\begin{gathered}
\phi_{i j}=\frac{1}{\vartheta \mu}\left|\frac{\Sigma_{i}}{\Gamma_{j}}\right| \\
\chi_{i j}=\operatorname{angle}\left(\frac{\Sigma_{i}}{\Gamma_{j}}\right)
\end{gathered}
$$

\subsection{Introduction of Cole-Cole Model}

In the actual electrical exploration work, the electromagnetic effect and induced polarization effect of the earth can often be approximated as a linear time-invariant system. The Cole-Cole model is the most commonly used to describe the induced polarization effect of rock and ore in this system. One of the mathematical models, the equivalent circuit is shown in Figure 1, where the sum of $R$ and $(j \vartheta \mu)^{-c}$ the polarization equivalent resistance, $c$ is a constant real number, and it is generally an underground resistance.

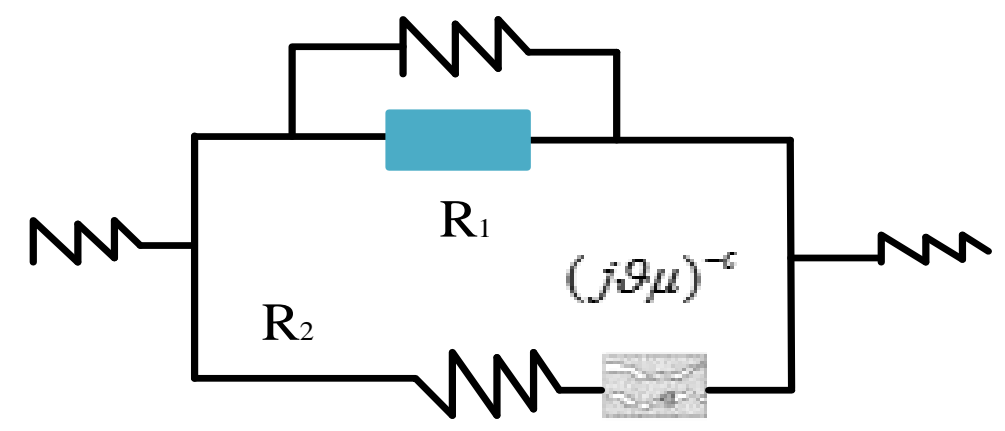

Fig.1 Schematic Diagram of the Equivalent Circuit of the Cole-Cole Model

The spectrum of the complex resistivity caused by the induced polarization effect in the ColeCole model can be expressed by formula (6). $\rho_{o}$ represents the resistivity when the frequency is zero; $p$ is the polarizability; $t$ is time constant, $c$ is the frequency correlation coefficient, and $c$ takes a value between 0 and 1 . According to the actual situation, the range of the remaining parameters in the complex resistivity formula is approximately:

$$
\rho(j \vartheta)=\rho_{o}\left[1-\lambda\left(1-\frac{1}{1+(j \vartheta \varpi)^{c}}\right)\right]
$$




\section{Analysis of Model Results}

In order to obtain the influence of different induced polarization parameters on the response of the MT two-dimensional electromagnetic field, two models are designed: one is a uniform halfspace model, and the other is a horizontal double prism model.

\subsection{Analysis of Response Characteristics under Different Polarizabilities}

The given true resistivity $\rho$ in the uniform half-space model is $145 \mathrm{~m}$, and the polarizabilities are: $p=0, p=0.2, p=0.4$. Using formula (6) can calculate the corresponding zero-frequency resistivity $\rho=150 \Omega \cdot m \quad \rho=165 \Omega \cdot m, \rho=175 \Omega \cdot m$, and the remaining parameters $\varpi=100$. $c=0.20$. The observation point is at the midpoint of the model. In TE and TM modes, the regular curve of apparent resistivity and apparent phase change with frequency in uniform half-space is shown in Figure 2.

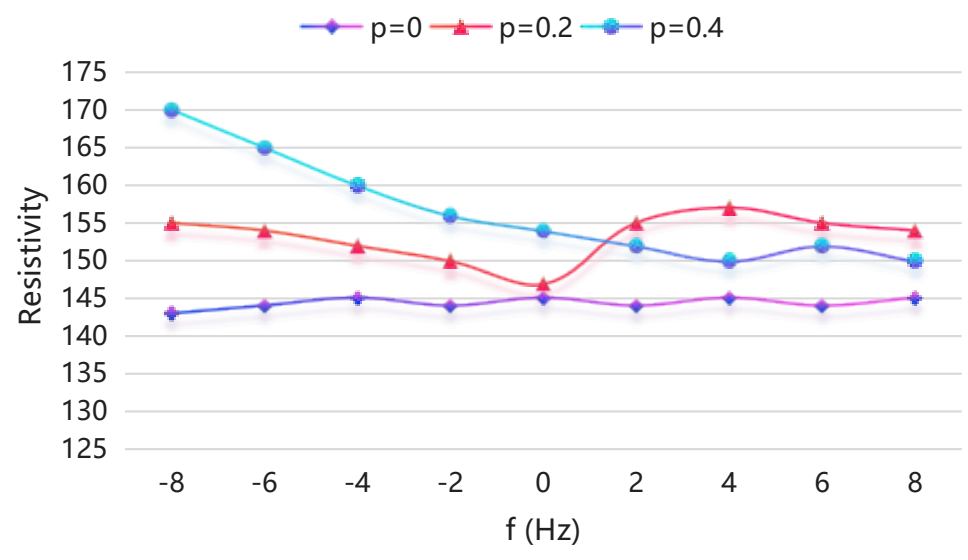

Fig.2 Curves of Apparent Resistivity and Apparent Phase Versus Frequency under Different Polarizabilities

It can be seen from Figure 2 that the apparent resistivity and apparent phase in TE mode are roughly equal to those in TM mode at low frequencies, but there is a certain deviation between the two modes at high frequencies. Generally speaking, there are many reasons for these deviations. The error in this trial calculation is mainly due to the truncation error[6]. In addition, it can be seen that the polarizability has a significant influence on the apparent resistivity: when the frequency is the same, the greater the polarizability, the greater the apparent resistivity. As the frequency increases, the apparent resistivity gradually decreases, and finally it is approximately equal to the true resistivity. The law represented by the apparent phase curve is roughly the same: within a certain frequency range, at a certain frequency, the greater the polarizability, the greater the value of the apparent phase.

The above phenomenon occurs because the impedance caused by the induced polarization effect has the nature of capacitive reactance. The lower the frequency, the stronger the impedance effect in the polarization area, and the greater the polarization equivalent impedance. According to the equivalent circuit diagram, the total impedance is smaller than Real impedance underground.

When $t \rightarrow \infty$ or $f \rightarrow 0$, the zero-frequency resistivity $\rho^{*}$ is equal to the limit equivalent resistivity equal to the ratio of the true resistivity, which is:

$$
\rho^{*}=\frac{\rho}{1-\delta}
$$

\subsection{Response Characteristics of Prism Model}

A schematic diagram of two polarized prisms placed horizontally in a uniform half-space. The 
angle between the survey line direction and the abnormal body structure direction is set to 0 or 100 . The background resistivity is $\rho=145 \Omega \cdot m$, the true resistivity of the left and right prisms is $5.6 \Omega \cdot \mathrm{m}, 2280 \Omega \cdot \mathrm{m}$, and the size of the study area is $10 \mathrm{~km} * 8 \mathrm{~km}$, and the buried depth of the anomalous body is $1 \mathrm{~km}$. Similarly, use formula (7), corresponding zero-frequency resistivity can be calculated. Calculate the curves of apparent resistivity and apparent phase in TM and TE modes when $f=0.2 \mathrm{~Hz}$, and the results are shown in Figure 3 .

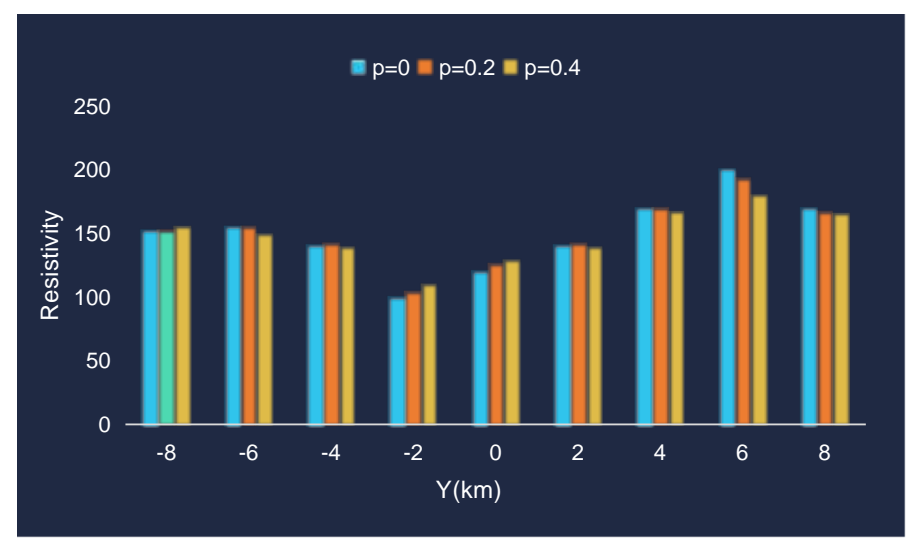

Fig.3 Curves of Influence of Different Polarizabilities on Apparent Resistivity and Apparent Phase in Tm Mode $(f=0.2 \mathrm{~Hz})$

It can be seen from the apparent resistivity curve in Figure 3 that the low-resistance and highresistance abnormalities can be reflected in the TM mode. However, the difference in the influence of the change of the polarization rate $\mathrm{m}$ is not big, and the difference between the apparent phases is not very big. This is because the greater the frequency $f=0.2 \mathrm{~Hz}$, the smaller the difference in apparent resistivity produced by different polarizabilities $\mathrm{m}$, and the presence of low-resistance bodies increases the apparent phase, and the presence of high-resistance bodies makes the apparent phase reduce.

The influence curve of the polarization rate change on the apparent resistivity and the apparent phase in the TE mode when the frequency is $0.2 \mathrm{~Hz}$. It can be seen that the TE mode is more sensitive to the change of the polarization rate $\mathrm{m}$, but in this mode, only It can reflect the lowresistance abnormal body, and the high-resistance abnormal body has almost no response[9]. At the same time, when the frequency is the same, both the apparent resistivity and the apparent phase increase with the increase of the polarizability, which is the same as the conclusion obtained in model 1 of this paper. The comparison of the two modes shows that the lateral resolution in the TM mode of the magnetotelluric sounding method is higher than that in the TE mode.

\subsection{The Influence of Resistivity on Response}

In order to study the influence of different complex resistivity parameters on apparent resistivity and apparent phase, the following discussion is carried out. Since the lateral resolution of the TM mode is higher than that of the TE mode, this discussion takes the TM mode as an example. In Figure 4 , the polarizability $\mathrm{p}=0.2, c=0.30$, change the time constant $\mathrm{T}$, the zero frequency resistivity is the same as above.

Figure 4 shows the influence curve of different complex resistivity parameters on apparent resistivity and apparent phase in TM mode. The study found that: complex resistivity parameters have an effect on the value of apparent resistivity and apparent phase, but different time constants and frequency correlation coefficients The difference between the apparent resistivity and the apparent phase is not much different, which can also be seen from formula (7). In comparison, the change of polarizability has a greater impact on apparent resistivity than other parameters. 


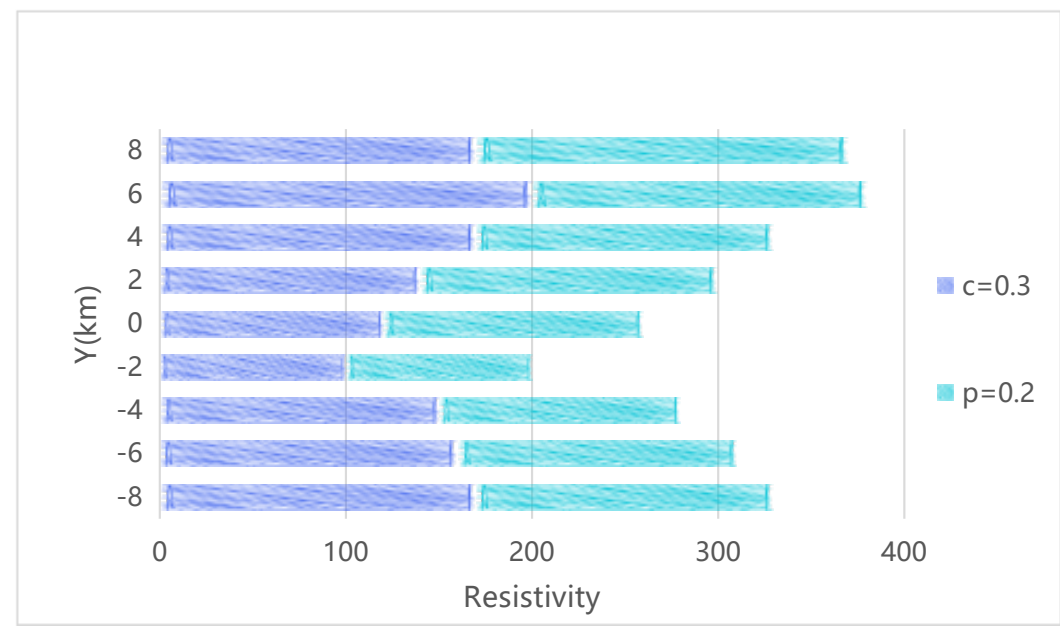

Fig.4 The Influence of Different Complex Resistivity Parameters on Apparent Resistivity and Apparent Phase $(f=0.2 \mathrm{~Hz})$

\section{Conclusion}

Through forward calculation, when there is an induced polarization phenomenon in the underground medium, the magnetotelluric sounding response contains the influence of complex resistivity parameters, and the induced polarization effect cannot be ignored. When the polarizability parameter becomes larger, the apparent resistivity above the polarization anomaly becomes larger and the apparent phase increases, but this change is not much different at high frequencies. When the abnormal body is polarized, both the apparent resistivity and the apparent phase increase compared with the case of no polarization. The change has the greatest impact, and this change is more pronounced at low frequencies; and the change in the time constant and frequency correlation coefficient does not have much effect on the apparent resistivity and apparent phase. Therefore, try to use a lower frequency when inverting the complex resistivity parameters.

\section{References}

[1] Payler, S.J., Biddle, J.F., Coates, A.J., Cousins, C.R., Cross, R.E., Cullen, D.C., Downs, M.T., Direito, S.O., Edwards, T., Gray, A.L. and Genis, J., "Planetary Science and Exploration in the Deep Subsurface: Results from the MINAR Program, Boulby Mine, UK.” International Journal of Astrobiology, vol. 16, no.2, pp.114-129,2016.

[2] Gong, S., Yang, Y., Lin, P., Wu, W., Zheng, C., Shi, F., Wu, X., Weng, A., Zhang, G., Gu, G. and Ye, Y. "Three - dimensional Electrical Exploration Methods for the Mapping of Polymetallic Targets in Gansu Province, China.” Geophysical Prospecting, vol.67,no.7, pp.1929-1947,2019.

[3] Taha, A., Ismail, A., Massoud, U., Mesbah, H. and Komarov, V. "Induced Polarization Measurement for Gold Exploration at the Estern Desert of Egupt.” Bulletin of Earth Sciences of Thailand,vol. 2, no.1, pp.20-30,2018.

[4] Song, H., Zhang, Y., Gao, J. and Zhang, Y. "Small Power ZVS Circuits for the MarineControlled Source Electromagnetic Transmitter.” The Journal of Engineering, vol.1, no.7,pp.43254330,2019.

[5] Wang, M., Deng, M., Wu, Z., Luo, X., Jing, J. and Chen, K.. “The Deep-tow Marine ControlledSource Electromagnetic Transmitter System for Gas Hydrate Exploration.” Journal of Applied Geophysics, vol.13,no.7, pp.138-144,2017.

[6] Cui, X., Tian, Z., Cui, S. and Jin, Q. “Development of a New Blasting Vibroseis Technique and its Application to the Exploration of Geological Structures.” International Journal of 
Technology, vol.8, no.5, pp.789-799,2017.

[7] Li, Y., Melo, A., Martinez, C. and Sun, J. "Geology Differentiation: A New Frontier in Quantitative Geophysical Interpretation in Mineral Exploration.” The Leading Edge, vol.38,no.1, pp.60-66,2019.

[8] Yongsheng, M., Xunyu, C. and Peirong, Z. "China's Shale Gas Exploration and Development: Understanding and Practice.” Petroleum Exploration and Development, vol.45, no.4, pp.589603,2018 .

[9] Benafan, O., Noebe, R.D. and Halsmer, T.J. "Static Rock Splitters based on High Temperature Shape Memory Alloys for Planetary Explorations.” Acta Astronautica, vol.1,no.18, pp.137157,2016 .

[10] Cheng, S., Wang, M., Deng, M., Chen, K. and Zhang, Q. “Test Analysis of High-Power Multifunction Borehole-Ground Electromagnetic Transmitting System under Field Conditions.” IEEE Access, vol. 1, no. 6, pp.74847-74853, 2018. 